\title{
FIGHTING FOR YOU
}

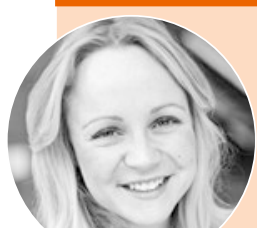

At the time of writing, the decision on whether graduation may be extended by universities if final year students are not deemed to be at a safe practising level and have not met requirements has not been made.

Having spoken with the Dental Schools Council (DSC) and COPDEND about how to optimise the chances for a timely graduation, we do recognise that students can only graduate if they demonstrate the necessary outcomes/competencies. However, we strongly believe that students should be given opportunity to graduate on time and progress to Dental Foundation Training (DFT) or Vocational Training (VT) and these positions should also be ready and available when students graduate. We will also push for funding for any additional academic fees and living costs for students if courses are extended.

These concerns play into a wider picture about clinical interaction that may affect students beyond 2021's graduating cohort. We know students are worried about the number of patients they are seeing. For example, some fourth and fifth years are getting no patient contact until at least January, whereas in other schools, some students have started AGP clinics and have been fit tested, whilst some are yet to do so.

Younger years have concerns as more priority for clinical time is placed on fourth and fifth years. There is a significant increase in the use of phantom heads to compensate, so students want to know if work on phantom heads would count towards their clinical targets. Again, we have discussed this with the DSC about how learning from dental schools offering best opportunities can be shared and applied elsewhere and provide consistency between universities.

With concerns about limited clinical exposure, we're aware that some students are not able to carry out procedures due to inadequate PPE. Protecting students - and staff - while maximising clinical contact is vital to your development. We have had reports of hoods being unavailable or not yet arrived and delayed PPE fittings. Some students have also received more training on correct use of PPE than others.

We have collated these reports from students and taken the results to the DSC. It is imperative all relevant guidelines are being followed to enable maximum clinical experience to be gained in a safe manner.

These measures will not last forever, but we know that right now they are vital to your safety and continued progression through dental school. We will continue to fight to ensure these aren't optional extras during your time at university because together, we are stronger.

Laura Assassa

\section{Looking for writing opportunities?}

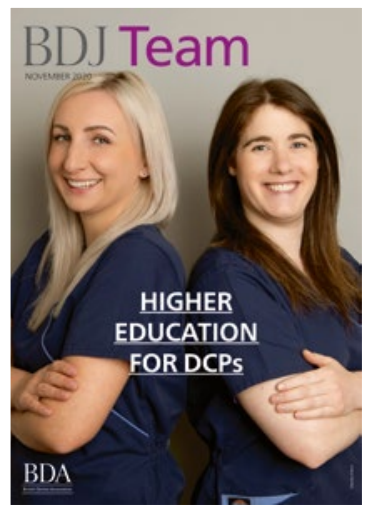

Would you like to write for the $B D J$ 's sister publication, $B D J$ Team?

$B D J$ Team is aimed at UK dental care professionals (DCPs) and published online ten times a year. The publication publishes a wide range of features, news, advice and informative content and is open to submissions or content ideas.

If you think you could write with a 'whole dental team' outlook and focus, there are

opportunities for publication in 2021.

To get a feel for the type of content published and discuss your content idea please visit www. nature.com/bdjteam and email k.quinlan@nature.com.

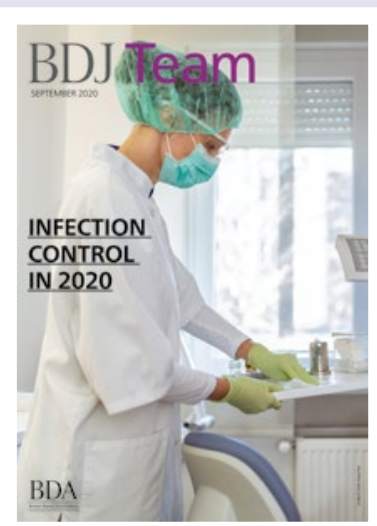

\section{STUDENT EDITOR WANTED}

If you're a budding writer, we have just the role for you. From Spring 2021, BDJ Student will be saying goodbye to current student editor, Sophia Antoniou, and require a new student editor from the Autumn issue onwards.

We're looking for someone with a passion for dentistry (obviously), a flair for writing, but above all we're looking for someone who can really bring together the voice of students. Previous editors have gone on to great heights - just ask current editor of $B D J$ Open,

Jonathan Lewney, who was student editor of Launchpad - BDJ Student's previous title back in the day!

All you need to do is submit a 500 word article you think would be suitable for BDJ Student on a topic that hasn't been covered in the last year before Friday 30 April to david.westgarth@bda. org to be considered.

Good luck! 